\title{
Epithelial dysregulation in obese severe asthmatics with gastro-oesophageal reflux
}

\author{
To the Editor:
}

Gastro-oesophageal reflux disease (GORD) and obesity are associated with frequent exacerbations and poor quality of life in people living with asthma. Multiple mechanisms have been proposed for the effect of obesity, including modification of inflammation affecting epithelial cell proliferation and wound repair, while the role of GORD is poorly understood and proton pump inhibitors (PPIs) are of variable efficacy. GORD might exert a deleterious effect by inducing vagal reflex, neuroinflammation and directly triggering airway inflammation (via microaspiration). Studies of reflux in animal models and human bronchial epithelial cell culture show varying impact on inflammation and airway remodelling. We have recently demonstrated changes in the sputum proteome in severe asthmatics with GORD, providing supportive evidence for gastric secretions exerting a direct effect on the airways [1]. The epithelium plays a key role in asthma, so in this study, we speculated that severe asthma in obese patients with GORD would be associated with epithelial dysfunction. Because GORD is treated with PPIs, drugs associated with risk of pneumonia and exacerbations of chronic obstructive pulmonary disease (COPD) and cystic fibrosis, the impact of PPIs was also assessed.

We analysed 61 never- or ex-smoker asthmatics and 44 healthy never-smokers from the U-BIOPRED (Unbiased Biomarkers in Prediction of Respiratory Disease Outcomes) study [2] who had undergone bronchoscopy, bronchial biopsy and epithelial brushing. Patients were categorised as obese if their body mass index was $\geqslant 30 \mathrm{~kg} \cdot \mathrm{m}^{-2}$, having or not a physician's diagnosis of GORD, and treated or not for GORD. Epithelial brushings were processed into RNAlater (Sigma-Aldrich, St Louis, MO, USA) for Affymetrix U133 Plus 2.0 (Affymetrix, Santa Clara, CA, USA) microarray analysis (GSE76226), and bronchial biopsies were immunostained for $\mathrm{CD}^{+}, \mathrm{CD}^{+}$and $\mathrm{CD}^{+}$lymphocytes, and analysed for basement membrane thickness. The study was approved by national ethics committees. All participants provided consent.

Epithelial transcriptomic data were clustered by topological data analysis (TDA) using the Ayasdi Core software (Ayasdi, MenloPark, CA, USA) with cluster boundaries defined by density using Morse theory [3]. Paired t-tests were applied to $\log _{2}$-transformed transcriptomic data. Clinical data were analysed by Kruskal-Wallis, Mann-Whitney U-tests or Student t-tests depending on data distribution. False discovery rate correction was applied to the differentially expressed genes (DEGs). Pathway signatures and upstream regulators were identified by Ingenuity Pathway Analysis (IPA) (QIAGEN, Redwood City, CA, USA). Potential drug impact on DEGs was identified by connectivity map (CMap) analysis of DEG signatures.

TDA produced three clusters of similar size (C1, C2 and C3), comprising 21 participants (34\%) in C1, 23 (38\%) in C2 and 22 (36\%) in C3 (figure 1). When compared to combined C2/3 clusters, C1 had a higher incidence of obesity ( $76 \%$ versus $47 \%, \mathrm{p}=0.02)$, GORD ( $85 \%$ versus $43 \%, \mathrm{p}=0.04)$ and GORD treatment ( $81 \%$ versus $36 \%, \mathrm{p}=0.004$ ), and $48 \%$ were obese and had a diagnosis of GORD and GORD treatment (compared to $10 \%$ in $\mathrm{C} 2 / \mathrm{C} 3, \mathrm{p}=0.0009$ ); this cluster was, therefore, termed the obesity-GORD-PPI treatment (OGP) phenotype. When compared to C2/3, the OGP cluster had lower blood eosinophil counts $(\mathrm{p}=0.007)$ but was similar in respect of corticosteroid treatment.

@ERSpublications

Stratification of severe asthma on the basis of bronchial epithelial gene expression identified a subset whose endotype is defined by marked epithelial dysregulation associated with obesity, gastrooesophageal reflux and proton pump inhibitors http://bit.ly/2EeKv23

Cite this article as: Perotin J-M, Schofield JPR, Wilson SJ, et al. Epithelial dysregulation in obese severe asthmatics with gastro-oesophageal reflux. Eur Respir J 2019; 53: 1900453 [https://doi.org/10.1183/ 13993003.00453-2019]. 

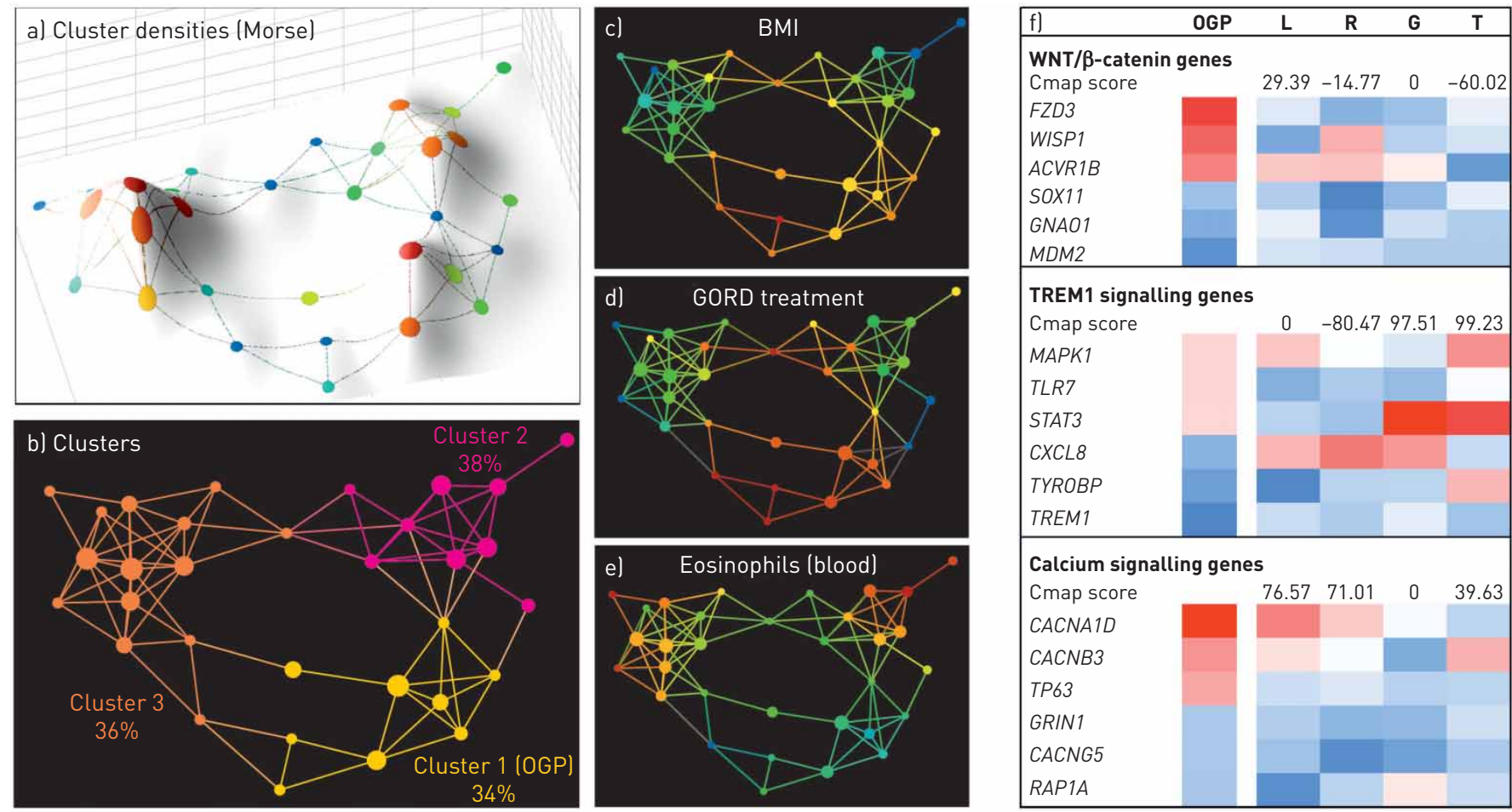
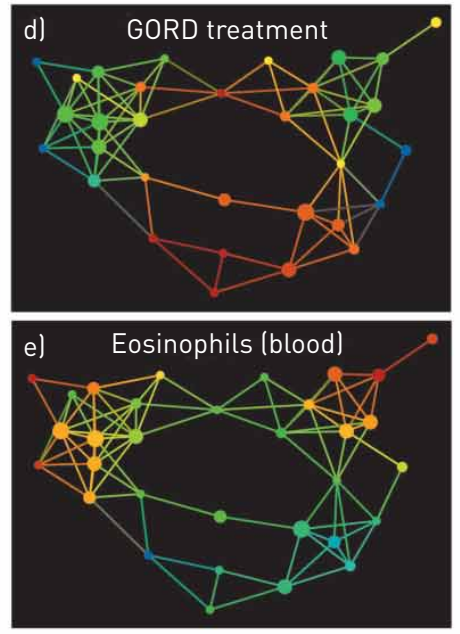

FIGURE 1 Severe asthma clusters based on epithelial transcriptomics. a) Topological data analysis network constructed with transcripts from bronchial brushings using density analysis by Morse theory, with b) the identified clusters 1-3 coloured yellow, pink and orange. Applying c) body mass index (BMI), d) gastro-oesophageal reflux disease (GORD) treatment and e) blood eosinophil counts as metadata, nodes are coloured by intensity from blue (low intensity) to red (high intensity). f) Heat map of the three top upregulated and three top downregulated differentially expressed genes of wingless tail (WNT)/ $\beta$-catenin, triggering receptor expressed on myeloid cells (TREM) 1 and calcium signalling in the obesityGORD-proton pump inhibitor treatment (OGP) cluster and in airway epithelial cells exposed to proton pump inhibitors (lansoprazole (L) and rabeprazole (R)) and bile acids (glycodeoxycholic acid (G) and taurodeoxycholic acid (T)). Cmap: connectivity mapping.

IPA identified 77 pathways dysregulated in the OGP cluster relative to health, the top being the wingless tail (WNT)/ $\beta$-catenin pathway (z-score: 2.2 -fold difference versus healthy participants). Amongst the 38 DEGs related to WNT/ $\beta$-catenin signalling, FZD3 (frizzled 2) and WISP1 (WNT1-inducible signalling pathway protein 1) were amongst the top upregulated genes (figure 1). Application of CMap analysis to these DEGs and comparison with the genes regulated by PPI and bile acids in A549 epithelial cells (figure 1) showed that the WNT/ $\beta$-catenin signalling pathway was not associated with PPI or bile acid effects. Furthermore, although evidence points to $\mathrm{WNT} / \beta$-catenin signalling and the WNT target gene WISP1 regulating airway remodelling and pulmonary myofibroblast proliferation in COPD and idiopathic pulmonary fibrosis [4], we did not find a difference in the thickness of the subepithelial lamina reticularis between the OGP cluster and the other patients.

WISP1 has been shown to be upregulated in obese individuals but has also been identified as an inhibitor of adipocyte differentiation by blocking the induction of the adipogenic transcription factors peroxisome proliferator-activated receptor (PPAR) $\gamma$ and CCAAT enhancer-binding protein $\alpha$ [5], both of which were downregulated in the OGP cluster. Given that PPAR $\gamma$ expression in bronchial epithelial cells has been shown to protect against oxidative stress and suppress mucin 5AC expression, these data suggested that the OGP phenotype should be associated with airway inflammation. However, our study showed that the OGP cluster had a predominantly paucigranulocytic sputum cell profile $(\mathrm{p}=0.017)$ and fewer submucosal T-cells when compared to health $\left(\mathrm{CD}^{+}\right.$cells: 10.2 versus 20.4 per $\mathrm{mm}^{2}, \mathrm{p}=0.05$; $\mathrm{CD} 4^{+}$cells: 7.2 versus 10.4 per $\mathrm{mm}^{2}$, $\mathrm{p}=0.02$; $\mathrm{CD}^{+}$cells: 23.0 versus 36.7 per $\mathrm{mm}^{2}, \mathrm{p}=0.03$ ). This could be explained by the finding in the OGP cluster of downregulated immune response pathways, including proliferation, activation and survival of lymphocytes, leukocyte recruitment and transepithelial migration (interleukin-7, triggering receptor expressed on myeloid cells (TREM)1), B-cell receptor and $\mathrm{Ca}^{2+}$ signalling; $\mathrm{z}$-scores $-0.69,-2.18,-2.33$ and -3.66 , respectively). When compared to health and C2/3, the OGP cluster also exhibited downregulation of C-C ligand (CCL)5, CXC ligand (CXCL)1 and CCL11. CMap analysis identified high connectivity scores between TREM1 and effects of bile acids (CMap score 99.2), and between PPI treatment and $\mathrm{Ca}^{2+}$ signalling (CMap score 76.6) (figure 1), suggesting a direct impact of bile acids and PPI treatment on immune cell accumulation. 
In support of our study, recent analysis of bile acid effects on lipopolysaccharide-stimulated macrophages identified downregulated genes involved in differentiation and migration of immune cells, including T-cells, and decreased chemokine expression, including CCL5 and CXCL1 [6]. PPI inhibition of $\mathrm{H}^{+} /$ $\mathrm{K}^{+}$-ATPase induces intracellular acidification that inhibits immune cell proliferation, decreases heparinase activation involved in extracellular matrix remodelling and degradation [7], and decreases intercellular adhesion molecule expression, resulting in reduced immune cell transmigration [8]. In airway epithelial cells, Toll-like receptor (TLR) 2 activation by damage-associated molecular patterns (DAMPs) releases $\mathrm{Ca}^{2+}$ from endoplasmic reticulum stores, resulting in chemokine regulation through activation of nuclear factor $\kappa$-light chain-enhancer of activated B-cells $(\mathrm{NF}-\kappa \mathrm{B})$ and calpains, which cleave junctional proteins and facilitate immune cell transmigration [9]. In our study, expression of TLR2, NF- $\kappa B$ and NF- $\kappa$ B-regulated chemokines (CCL5, CXCL1, CXCL2, CCL11, CCL22 and CXCL8) was also downregulated in the OGP cluster. In contrast, expression of calreticulin and calnexin, two endoplasmic reticulum $\mathrm{Ca}^{2+}$ storage proteins, whose expression is increased by low intracellular $\mathrm{Ca}^{2+}$ storage [10], was upregulated in the OGP cluster, suggesting dysregulated intracellular $\mathrm{Ca}^{2+}$ influx. This could be speculated to involve PPI-induced decrease of $\mathrm{Ca}^{2+}$-ATPase sensitivity as previously shown in myocytes [11].

The top upstream regulator for the OGP cluster was CD24 (activation z-score 2.2, p=0.0004), a cell surface receptor involved in suppression of immune responses to DAMPs [12], organisation of tight junction proteins [13], regulation of adipogenesis, B-cell survival and T-cell activity [14]. CD24 is a direct WNT target gene [15], which is consistent with our findings of upregulated WNT/ $\beta$-catenin signalling. Together, these associations suggest a central role for CD24 in the OGP phenotype.

In summary, this exploratory analysis of epithelial gene expression in severe asthma has identified three clusters, one of which is enriched for obesity, GORD and treatment with PPIs, with an as yet unreported mechanism that could represent a new endotype. This potentially new endotype was shown to be paucigranulocytic as a consequence of downregulated mechanisms of cell recruitment linked to bile acid exposure and PPI treatment. The implications of this endotype for virus-induced exacerbations, which are increased in asthmatics with GORD, remains to be elucidated.

Jeanne-Marie Perotin ${ }^{1}$, James P.R. Schofield ${ }^{1,2}$, Susan J. Wilson ${ }^{3}$, Jonathan Ward $^{3}$, Joost Brandsma ${ }^{1}$, Fabio Strazzeri ${ }^{4}$, Aruna Bansal ${ }^{5}$, Xian Yang $^{6}$, Anthony Rowe ${ }^{7}$, Julie Corfield ${ }^{8}$, Rene Lutter, ${ }^{9,10}$, Dominick E. Shaw $\oplus^{11}$, Per S. Bakke ${ }^{12}$, Massimo Caruso $\oplus^{13,14}$, Barbro Dahlén ${ }^{15}$, Stephen J. Fowler $\oplus^{16}$, Ildikó Horváth ${ }^{17}$, Peter Howarth ${ }^{1}$, Norbert Krug $^{18}$, Paolo Montuschi ${ }^{19}$, Marek Sanak ${ }^{20}$, Thomas Sandström ${ }^{21}$, Kai Sun ${ }^{7}$, Ioannis Pandis ${ }^{7}$, Charles Auffray ${ }^{22}$, Bertrand De Meulder ${ }^{22}$, Diane Lefaudeux $\circledast^{22}$, John H. Riley ${ }^{23}$, Ana R. Sousa ${ }^{23}$, Sven-Erik Dahlen ${ }^{24}$, Ian M. Adcock ${ }^{25}$, Kian Fan Chung ${ }^{25}$, Peter J. Sterk ${ }^{11}$, Paul J. Skipp ${ }^{2}$, Jane E. Collins ${ }^{1}$, Donna E. Davies ${ }^{1}$ and Ratko Djukanović ${ }^{1}$ on behalf of the U-BIOPRED Study Group ${ }^{26}$

${ }^{1}$ NIHR Southampton Biomedical Research Centre, Clinical and Experimental Sciences, Faculty of Medicine, University of Southampton, Southampton, UK. ${ }^{2}$ Centre for Proteomic Research, Biological Sciences, University of Southampton, Southampton, UK. ${ }^{3}$ The Histochemistry Research Unit, Faculty of Medicine, University of Southampton, Southampton, UK. ${ }^{4}$ Mathematical Sciences, University of Southampton, Southampton, UK. ${ }^{5}$ Acclarogen Ltd, Cambridge, UK. ${ }^{6}$ Data Science Institute, Imperial College London, London, UK. ${ }^{7}$ Janssen Research and Development, High Wycombe, UK.

${ }^{8}$ Areteva Ltd, Nottingham, UK. ${ }^{9}$ Amsterdam UMC, Dept of Experimental Immunology (Amsterdam Infection and Immunity Institute), University of Amsterdam, Amsterdam, The Netherlands. ${ }^{10}$ Amsterdam UMC, Dept of Respiratory Medicine, University of Amsterdam, Amsterdam, The Netherlands. ${ }^{11}$ NIHR Biomedical Respiratory Research Centre, University of Nottingham, Nottingham, UK. ${ }^{12}$ Institute of Medicine, University of Bergen, Bergen, Norway. ${ }^{13}$ Dept of Clinical and Experimental Medicine Hospital University, University of Catania, Catania, Italy. ${ }^{14}$ Dept of Biomedical and Biotechnological Sciences (Biometec), University of Catania, Catania, Italy. ${ }^{15}$ Dept of Respiratory Diseases and Allergy, Karolinska University Hospital, Karolinska Institutet, Stockholm, Sweden. ${ }^{16}$ Respiratory and Allergy Research Group, University of Manchester, Manchester, UK. ${ }^{17}$ Dept of Pulmonology, Semmelweis University, Budapest, Hungary.

${ }^{18}$ Fraunhofer Institute for Toxicology and Experimental Medicine Hannover, Hannover, Germany. ${ }^{19}$ Faculty of Medicine, Catholic University of the Sacred Heart, Fondazione Policlinico Universitario, Agostino Gemelli IRCCS, Rome, Italy. ${ }^{20}$ Laboratory of Molecular Biology and Clinical Genetics, Medical College, Jagiellonian University, Krakow, Poland. ${ }^{21}$ Dept of Medicine, Dept of Public Health and Clinical Medicine Respiratory Medicine Unit, Umeå University, Umeå, Sweden. ${ }^{22}$ European Institute for Systems Biology and Medicine, CNRS-ENS-UCBL-INSERM, Université de Lyon, Lyons, France. ${ }^{23}$ Respiratory Therapeutic Unit, GSK, Uxbridge, UK. ${ }^{24}$ The Centre for Allergy Research, The Institute of Environmental Medicine, Karolinska Institutet, Stockholm, Sweden. ${ }^{25}$ Cell and Molecular Biology Group, Airways Disease Section, National Heart and Lung Institute, Imperial College London, London, UK. ${ }^{26}$ Collaborators in the U-BIOPRED Study Group are listed in the acknowledgements section.

Correspondence: Jeanne-Marie Perotin, NIHR Southampton Biomedical Research Centre, Clinical and Experimental Sciences, Faculty of Medicine, University of Southampton, mailpoint 810, Level F, Sir Henry Wellcome Laboratories, South Block, Southampton University Hospital, Southampton, SO16 6YD, UK. E-mail: j-m.perotin-collard@soton.ac.uk

Received: Dec 172018 | Accepted after revision: March 202019

Acknowledgements: J-M. Perotin is the recipient of a European Respiratory Society Long-Term Fellowship (LTRF 2017) and received additional funding to support her research in Southampton, UK, from the Association Régionale pour l'Aide aux Insuffisants Respiratoires de Champagne-Ardenne, Association Nationale de Formation Continue en Allergologie, Association des Allergologues de Champagne-Ardenne and Association des Pneumologues de Champagne-Ardenne. This funding is gratefully acknowledged. 
The U-BIOPRED consortium wishes to acknowledge the help and expertise of the following individuals and groups without whom the study would not have been possible: I.M. Adcock, National Heart and Lung Institute, Imperial College, London, UK; H. Ahmed, European Institute for Systems Biology and Medicine, CNRS-ENS-UCBL-INSERM, Lyon, France; C. Auffray, European Institute for Systems Biology and Medicine, CNRS-ENS-UCBL-INSERM, Lyon, France; P. Bakke, Dept of Clinical Science, University of Bergen, Bergen, Norway; A.T. Banssal, Acclarogen Ltd, St John's Innovation Centre, Cambridge, UK; F. Baribaud, Janssen R\&D, USA; S. Bates, Respiratory Therapeutic Unit, GSK, London, UK; E.H. Bel, Academic Medical Centre, University of Amsterdam, Amsterdam, The Netherlands; J. Bigler, previously Amgen Inc.; H. Bisgaard, COPSAC, Copenhagen Prospective Studies on Asthma in Childhood, Herlev and Gentofte Hospital, University of Copenhagen, Copenhagen, Denmark; M.J. Boedigheimer, Amgen Inc., Thousand Oaks, USA; K. Bønnelykke, COPSAC, Copenhagen Prospective Studies on Asthma in Childhood, Herlev and Gentofte Hospital, University of Copenhagen, Copenhagen, Denmark; J. Brandsma, University of Southampton, Southampton, UK; P. Brinkman, Academic Medical Centre, University of Amsterdam, Amsterdam, The Netherlands; E. Bucchioni, Chiesi Pharmaceuticals SPA, Parma, Italy; D. Burg, Centre for Proteomic Research, Institute for Life Sciences, University of Southampton, Southampton, UK; A. Bush, National Heart and Lung Institute, Imperial College, London, UK; Royal Brompton and Harefield NHS trust, UK; M. Caruso, Dept Clinical and Experimental Medicine, University of Catania, Catania, Italy; A. Chaiboonchoe, European Institute for Systems Biology and Medicine, CNRS-ENS-UCBL-INSERM, Lyon, France; P. Chanez, Assistance publique des Hôpitaux de Marseille - Clinique des bronches, allergies et sommeil, Aix Marseille Université, Marseille, France; K.F. Chung, National Heart and Lung Institute, Imperial College, London, UK; C.H. Compton, Respiratory Therapeutic Unit, GSK, London, UK; J. Corfield, Areteva R\&D, Nottingham, UK; A. D'Amico, University of Rome "Tor Vergata", Rome Italy; S.E. Dahlen, Centre for Allergy Research, Karolinska Institutet, Stockholm, Sweden; B. De Meulder, European Institute for Systems Biology and Medicine, CNRS-ENS-UCBL-INSERM, Lyon, France; R. Djukanovic, NIHR Southampton Respiratory Biomedical Research Unit and Clinical and Experimental Sciences, Southampton, UK; V.J. Erpenbeck, Translational Medicine, Respiratory Profiling, Novartis Institutes for Biomedical Research, Basel, Switzerland; D. Erzen, Boehringer Ingelheim Pharma GmbH \& Co. KG, Biberach, Germany; K. Fichtner, Boehringer Ingelheim Pharma GmbH \& Co. KG, Biberach, Germany; N. Fitch, BioSci Consulting, Maasmechelen, Belgium; L.J. Fleming, National Heart and Lung Institute, Imperial College, London, UK; Royal Brompton and Harefield NHS trust, UK; E. Formaggio, previously CROMSOURCE, Verona, Italy; S.J. Fowler, Centre for Respiratory Medicine and Allergy, Institute of Inflammation and Repair, University of Manchester and University Hospital of South Manchester, Manchester Academic Health Sciences Centre, Manchester, UK; U. Frey, University Children's Hospital, Basel, Switzerland; M. Gahlemann, Boehringer Ingelheim (Schweiz) GmbH, Basel, Switzerland; T. Geiser, Dept of Respiratory Medicine, University Hospital Bern, Switzerland; Y. Guo, Data Science Institute, Imperial College, London, UK; S. Hashimoto, Academic Medical Centre, University of Amsterdam, Amsterdam, The Netherlands; J. Haughney, International Primary Care Respiratory Group, Aberdeen, UK; G. Hedlin, Dept Women's and Children's Health \& Centre for Allergy Research, Karolinska Institutet, Stockholm, Sweden; P.W. Hekking, Academic Medical Centre, University of Amsterdam, Amsterdam, The Netherlands, T. Higenbottam, Allergy Therapeutics, West Sussex, UK; J.M. Hohlfeld, Fraunhofer Institute for Toxicology and Experimental Medicine, Hannover, Germany; C. Holweg, Respiratory and Allergy Diseases, Genentech, San Francisco, USA; I. Horváth, Semmelweis University, Budapest, Hungary; P. Howarth, NIHR Southampton Respiratory Biomedical Research Unit, Clinical and Experimental Sciences and Human Development and Health, Southampton, UK; A.J. James, Centre for Allergy Research, Karolinska Institutet, Stockholm, Sweden; R. Knowles, Arachos Pharma, Stevenge, UK; A.J. Knox, Respiratory Research Unit, University of Nottingham, Nottingham, UK; N. Krug, Fraunhofer Institute for Toxicology and Experimental Medicine, Hannover, Germany; D. Lefaudeux, European Institute for Systems Biology and Medicine, CNRS-ENS-UCBL-INSERM, Lyon, France; M.J. Loza, Janssen R\&D, USA; R. Lutter, Academic Medical Centre, University of Amsterdam, Amsterdam, The Netherlands; A. Manta, Roche Diagnostics GmbH, Mannheim, Germany; S. Masefield, European Lung Foundation, Sheffield, UK; J.G. Matthews, Respiratory and Allergy Diseases, Genentech, San Francisco, USA; A. Mazein, European Institute for Systems Biology and Medicine, CNRS-ENS-UCBL-INSERM, Lyon, France; A. Meiser, Data Science Institute, Imperial College, London, UK; R.J.M. Middelveld, Centre for Allergy Research, Karolinska Institutet, Stockholm, Sweden; M. Miralpeix, Almirall, Barcelona, Spain; P. Montuschi, Università Cattolica del Sacro Cuore, Milan, Italy; N. Mores, Università Cattolica del Sacro Cuore, Milan, Italy; C.S. Murray, Centre for Respiratory Medicine and Allergy, Institute of Inflammation and Repair, University of Manchester and University Hospital of South Manchester, Manchester Academic Health Sciences Centre, Manchester, UK; J. Musial, Dept of Medicine, Jagiellonian University Medical College, Krakow, Poland; D. Myles, Respiratory Therapeutic Unit, GSK, London, UK; L. Pahus, Assistance publique des Hôpitaux de Marseille, Clinique des bronches, allergies et sommeil, Espace Éthique Méditerranéen, Aix-Marseille Université, Marseille, France; I. Pandis, Data Science Institute, Imperial College, London, UK; S. Pavlidis, National Heart and Lung Institute, Imperial College, London, UK; P. Powell, European Lung Foundation, Sheffield, UK; G. Praticò, CROMSOURCE, Verona, Italy; M. Puig Valls, CROMSOURCE, Barcelona, Spain; N. Rao, Janssen R\&D, USA; J. Riley, Respiratory Therapeutic Unit, GSK, London, UK; A. Roberts, Asthma UK, London, UK; G. Roberts, NIHR Southampton Respiratory Biomedical Research Unit, Clinical and Experimental Sciences and Human Development and Health, Southampton, UK; A. Rowe, Janssen R\&D, UK; T. Sandström, Dept of Public Health and Clinical Medicine, Umeå University, Umeå, Sweden; W. Seibold, Boehringer Ingelheim Pharma GmbH, Biberach, Germany; A. Selby, NIHR Southampton Respiratory Biomedical Research Unit, Clinical and Experimental Sciences and Human Development and Health, Southampton, UK; D.E. Shaw, Respiratory Research Unit, University of Nottingham, UK; R. Sigmund, Boehringer Ingelheim Pharma GmbH \& Co. KG, Biberach, Germany; F. Singer, University Children’s Hospital, Zurich, Switzerland; P.J. Skipp, Centre for Proteomic Research, Institute for Life Sciences, University of Southampton, Southampton, UK; A.R. Sousa, Respiratory Therapeutic Unit, GSK, London, UK; P.J. Sterk, Academic Medical Centre, University of Amsterdam, Amsterdam, The Netherlands; K. Sun, Data Science Institute, Imperial College, London, UK; B. Thornton, MSD, USA W.M. van Aalderen, Academic Medical Centre, University of Amsterdam, Amsterdam, The Netherlands; M. van Geest, AstraZeneca, Mölndal, Sweden; J. Vestbo, Centre for Respiratory Medicine and Allergy, Institute of Inflammation and Repair, University of Manchester and University Hospital of South Manchester, Manchester Academic Health Sciences Centre, Manchester, UK; N.H. Vissing, COPSAC, Copenhagen Prospective Studies on Asthma in Childhood, Herlev and Gentofte Hospital, University of Copenhagen, Copenhagen, Denmark; A.H. Wagener, Academic Medical Center Amsterdam, Amsterdam, The Netherlands; S.S. Wagers, BioSci Consulting, Maasmechelen, Belgium; Z. Weiszhart, 
Semmelweis University, Budapest, Hungary; C.E. Wheelock, Centre for Allergy Research, Karolinska Institutet, Stockholm, Sweden; S.J. Wilson, Histochemistry Research Unit, Faculty of Medicine, University of Southampton, Southampton, UK. Additional contributors: Antonios Aliprantis, Merck Research Laboratories, Boston, USA; David Allen, North West Severe Asthma Network, Pennine Acute Hospital NHS Trust, UK; Kjell Alving, Dept Women's \& Children's Health, Uppsala University, Uppsala, Sweden; P. Badorrek, Fraunhofer ITEM, Hannover, Germany; David Balgoma, Centre for Allergy Research, Karolinska Institutet, Stockholm, Sweden; S. Ballereau, European institute for Systems Biology and Medicine, University of Lyon, France; Clair Barber, NIHR Southampton Respiratory Biomedical Research Unit and Clinical and Experimental Sciences, Southampton, UK; Manohara Kanangana Batuwitage, Data Science Institute, Imperial College, London, UK; An Bautmans, MSD, Brussels, Belgium; A. Bedding, Roche Diagnostics $\mathrm{GmbH}$, Mannheim, Germany; A.F. Behndig, Umeå University, Umea, Sweden; Jorge Beleta, Almirall S.A., Barcelona, Spain; A. Berglind, MSD, Brussels, Belgium; A. Berton, AstraZeneca, Mölndal, Sweden; G. Bochenek, II Dept of Internal Medicine, Jagiellonian University Medical College, Krakow, Poland; A. Braun, Fraunhofer Institute for Toxicology and Experimental Medicine, Hannover, Germany; D. Campagna, Dept of Clinical and Experimental Medicine, University of Catania, Catania, Italy; L. Carayannopoulos, previously at MSD, USA; C. Casaulta, University Children's Hospital of Bern, Switzerland; Romanas Chaleckis, Centre of Allergy Research, Karolinska Institutet, Stockholm, Sweden; B. Dahlén, Karolinska University Hospital \& Centre for Allergy Research, Karolinska Institutet, Stockholm, Sweden; T. Davison, Janssen R\&D, USA; J. De Alba, Almirall S.A., Barcelona, Spain; I. De Lepeleire, MSD, Brussels, Belgium; T. Dekker, Academic Medical Centre, University of Amsterdam, Amsterdam, The Netherlands; I. Delin, Centre for Allergy Research, Karolinska Institutet, Stockholm, Sweden; P. Dennison, NIHR Southampton Respiratory Biomedical Research Unit, Clinical and Experimental Sciences, NIHR-Wellcome Trust Clinical Research Facility, Faculty of Medicine, University of Southampton, Southampton, UK; A. Dijkhuis, Academic Medical Centre, University of Amsterdam, Amsterdam, The Netherlands; P. Dodson, AstraZeneca, Mölndal, Sweden; Aleksandra Draper, BioSci Consulting, Maasmechelen, Belgium; K. Dyson, CROMSOURCE, Stirling, UK; J. Edwards, Asthma UK, London, UK; L. El Hadjam, European Institute for Systems Biology and Medicine, University of Lyon, France; R. Emma, Dept of Clinical and Experimental Medicine, University of Catania, Catania, Italy; M. Ericsson, Karolinska University Hospital, Stockholm, Sweden; C. Faulenbach, Fraunhofer ITEM, Hannover, Germany; Breda Flood, European Federation of Allergy and Airways Diseases Patient's Associations, Brussels, Belgium; G. Galffy, Semmelweis University, Budapest, Hungary; H. Gallart, Centre for Allergy Research, Karolinska Institutet, Stockholm, Sweden; D. Garissi, Global Head Clinical Research Division, CROMSOURCE, Italy; J. Gent, Royal Brompton and Harefield NHS Foundation Trust, London, UK; M. Gerhardsson de Verdier, AstraZeneca; Mölndal, Sweden; D. Gibeon, National Heart and Lung Institute, Imperial College, London, UK; Cristina Gomez, Centre for Allergy Research, Karolinska Institutet, Stockholm, Sweden; K. Gove, NIHR Southampton Respiratory Biomedical Research Unit and Clinical and Experimental Sciences, Southampton, UK; Neil Gozzard, UCB, Slough, UK; E. Guillmant-Farry, Royal Brompton Hospital, London, UK; E. Henriksson, Karolinska University Hospital \& Karolinska Institutet, Stockholm, Sweden; L. Hewitt, NIHR Southampton Respiratory Biomedical Research Unit, Southampton, UK; U. Hoda, Imperial College, London, UK; Richard Hu, Amgen Inc. Thousand Oaks, USA; S. Hu, National Heart and Lung Institute, Imperial College, London, UK; X. Hu, Amgen Inc., Thousand Oaks, USA; E. Jeyasingham, UK Clinical Operations, GSK, Stockley Park, UK; K. Johnson, Centre for Respiratory Medicine and Allergy, Institute of Inflammation and repair, University Hospital of South Manchester, NHS Foundation Trust, Manchester, UK; N. Jullian, European Institute for Systems Biology and Medicine, University of Lyon, France; J. Kamphuis, Longfonds, Amersfoort, The Netherlands; E.J. Kennington, Asthma UK, London, UK; D. Kerry, CromSource, Stirling, UK; G. Kerry, Centre for Respiratory Medicine and Allergy, Institute of Inflammation and Repair, University Hospital of South Manchester, NHS Foundation Trust, Manchester, UK; M. Klüglich, Boehringer Ingelheim Pharma GmbH \& Co. KG, Biberach, Germany; H. Knobel, Philips Research Laboratories, Eindhoven, The Netherlands; Johan Kolmert, Centre for Allergy Research, Karolinska Institutet, Stockholm, Sweden; J.R. Konradsen, Dept Women's and Children's Health \& Centre for Allergy Research, Karolinska Institutet, Stockholm, Sweden; M. Kots, Chiesi Pharmaceuticals, SPA, Parma, Italy; Kosmas Kretsos, UCB, Slough, UK; L. Krueger, University Children's Hospital Bern, Switzerland; S. Kuo, National Heart and Lung Institute, Imperial College, London, UK; M. Kupczyk, Centre for Allergy Research, Karolinska Institutet, Stockholm, Sweden; B. Lambrecht, University of Gent, Gent, Belgium; A-S. Lantz, Karolinska University Hospital \& Centre for Allergy Research, Karolinska Institutet, Stockholm, Sweden; Christopher Larminie, GSK, London, UK; L.X. Larsson, AstraZeneca, Mölndal, Sweden; P. Latzin, University Children's Hospital of Bern, Bern, Switzerland; N. Lazarinis, Karolinska University Hospital \& Karolinska Institutet, Stockholm, Sweden; N. Lemonnier, European Institute for Systems Biology and Medicine, CNRS-ENS-UCBL-INSERM, Lyon, France; S. Lone-Latif, Academic Medical Centre, University of Amsterdam, Amsterdam, The Netherlands; L.A. Lowe, Centre for Respiratory Medicine and Allergy, Institute of Inflammation and Repair, University Hospital of South Manchester, NHS Foundation Trust, Manchester, UK; A. Manta, Roche Diagnostics GmbH, Mannheim, Germany; L. Marouzet, NIHR Southampton Respiratory Biomedical Research Unit, Southampton, UK; J. Martin, NIHR Southampton Respiratory Biomedical Research Unit, Southampton, UK; C. Mathon, Centre of Allergy Research, Karolinska Institutet, Stockholm, Sweden; L. McEvoy, University Hospital, Dept of Pulmonary Medicine, Bern, Switzerland; S. Meah, National Heart and Lung Institute, Imperial College, London, UK; A. Menzies-Gow, Royal Brompton and Harefield NHS Foundation Trust, London, UK; L. Metcalf, previously at Asthma UK, London, UK; M. Mikus, Science for Life Laboratory \& The Royal Institute of Technology, Stockholm, Sweden; P. Monk, Synairgen Research Ltd, Southampton, UK; S. Naz, Centre for Allergy Research, Karolinska Institutet, Stockholm, Sweden; K. Nething, Boehringer Ingelheim Pharma GmbH \& Co. KG, Biberach, Germany; B. Nicholas, University of Southampton, Southampton, UK; U. Nihlén, previously at AstraZeneca, Mölndal, Sweden; Peter Nilsson, Science for Life Laboratory \& The Royal Institute of Technology, Stockholm, Sweden; R. Niven, North West Severe Asthma Network, University Hospital South Manchester, UK; B. Nordlund, Dept Women's and Children's Health \& Centre for Allergy Research, Karolinska Institutet, Stockholm, Sweden; S. Nsubuga, Royal Brompton Hospital, London, UK; J. Östling, AstraZeneca, Mölndal, Sweden; A. Pacino, Lega Italiano Anti Fumo, Catania, Italy; S. Palkonen, European Federation of Allergy and Airways Diseases Patient's Associations, Brussels, Belgium; J. Pellet, European Institute for Systems Biology and Medicine, CNRS-ENS-UCBL-INSERM, Lyon, France; G. Pennazza, University of Rome 'Tor Vergata', Rome Italy; A. Petrén, Centre for Allergy Research, Karolinska Institutet, Stockholm, Sweden; S. Pink, NIHR Southampton Respiratory Biomedical Research Unit, Southampton, UK; C. Pison, European Institute for Systems Biology and Medicine, CNRS-ENS-UCBL-INSERM, Lyon, France; A. Postle, University of Southampton, UK; M. Rahman-Amin, previously at Asthma UK, London, UK; L. Ravanetti, Academic Medical Centre, University of Amsterdam, Amsterdam, 
The Netherlands; E. Ray, NIHR Southampton Respiratory Biomedical Research Unit, Southampton, UK; S. Reinke, Centre for Allergy Research, Karolinska Institutet, Stockholm, Sweden; L. Reynolds, previously at Asthma UK, London, UK; K. Riemann, Boehringer Ingelheim Pharma GmbH \& Co. KG, Biberach, Germany; Martine Robberechts, MSD, Brussels, Belgium; J.P. Rocha, Royal Brompton and Harefield NHS Foundation Trust, UK; C. Rossios, National Heart and Lung Institute, Imperial College, London, UK; K. Russell, National Heart and Lung Institute, Imperial College, London, UK; M. Rutgers, Longfonds, Amersfoort, The Netherlands; G. Santini, Università Cattolica del Sacro Cuore, Milan, Italy; M. Santoninco, University of Rome "Tor Vergata", Rome Italy; M. Saqi, European Institute for Systems Biology and Medicine, CNRS-ENS-UCBL-INSERM, Lyon, France; C. Schoelch, Boehringer Ingelheim Pharma GmbH \& Co. KG, Biberach, Germany; J.P.R. Schofield, Centre for Proteomic Research, Institute for Life Sciences, University of Southampton, Southampton, UK; S. Scott, North West Severe Asthma Network, Countess of Chester Hospital, UK; N. Sehgal, North West Severe Asthma Network, Pennine Acute Hospital NHS Trust; M. Sjödin, Centre for Allergy Research, Karolinska Institutet, Stockholm, Sweden; B. Smids, Academic Medical Centre, University of Amsterdam, Amsterdam, The Netherlands; Caroline Smith, NIHR Southampton Respiratory Biomedical Research Unit, Southampton, UK; J. Smith, Asthma UK, London, UK; K.M. Smith, University of Nottingham, UK; P. Söderman, Dept Women's and Children's Health, Karolinska Institutet, Stockholm, Sweden; A. Sogbessan, Royal Brompton and Harefield NHS Foundation Trust, London, UK; F. Spycher, University Hospital Dept of Pulmonary Medicine, Bern, Switzerland; D. Staykova, University of Southampton, Southampton, UK; S. Stephan, Centre for Respiratory Medicine and Allergy, Institute of Inflammation and Repair, University Hospital of South Manchester, NHS Foundation Trust, Manchester, UK; J. Stokholm, University of Copenhagen and Danish Pediatric Asthma Centre Denmark; K. Strandberg, Karolinska University Hospital \& Karolinska Institutet, Stockholm, Sweden; M. Sunther, Centre for Respiratory Medicine and Allergy, Institute of Inflammation and Repair, University Hospital of South Manchester, NHS Foundation Trust, Manchester, UK; M. Szentkereszty, Semmelweis University, Budapest, Hungary; L. Tamasi, Semmelweis University, Budapest, Hungary; K. Tariq, NIHR Southampton Respiratory Biomedical Research Unit, Clinical and Experimental Sciences, NIHR-Wellcome Trust Clinical Research Facility, Faculty of Medicine, University of Southampton, Southampton, UK; J-O. Thörngren, Karolinska University Hospital, Stockholm, Sweden; Jonathan Thorsen, COPSAC, Copenhagen Prospective Studies on Asthma in Childhood, Herlev and Gentofte Hospital, University of Copenhagen, Copenhagen, Denmark; S. Valente, Università Cattolica del Sacro Cuore, Milan, Italy; Marianne van de Pol, Academic Medical Centre, University of Amsterdam, Amsterdam, The Netherlands; C.M. van Drunen, Academic Medical Centre, University of Amsterdam, Amsterdam, The Netherlands; J. Van Eyll, UCB, Slough, UK; J. Versnel, previously at Asthma UK, London, UK; A. Vink, Philips Research Laboratories, Eindhoven, The Netherlands; C. von Garnier, University Hospital Bern, Switzerland; A. Vyas, North West Severe Asthma Network, Lancashire Teaching Hospitals NHS Trust, UK; F. Wald, Boehringer Ingelheim Pharma GmbH \& Co. KG, Biberach, Germany; S. Walker, Asthma UK, London, UK; J. Ward, Histochemistry Research Unit, Faculty of Medicine, University of Southampton, Southampton, UK; K. Wetzel, Boehringer Ingelheim Pharma GmbH, Biberach, Germany; C. Wiegman, National Heart and Lung Institute, Imperial College, London, UK; S. Williams, International Primary Care Respiratory Group, Aberdeen, UK; X. Yang, Data Science Institute, Imperial College, London, UK; E. Yeyasingham, UK Clinical Operations, GSK, Stockley Park, UK; W. Yu, Amgen Inc., Thousand Oaks, USA; W. Zetterquist, Dept Women's and Children's Health \& Centre for Allergy Research, Karolinska Institutet, Stockholm, Sweden; Z. Zolkipli, NIHR Southampton Respiratory Biomedical Research Unit, Clinical and Experimental Sciences and Human Development and Health, Southampton, UK; A.H. Zwinderman, Academic Medical Centre, University of Amsterdam, The Netherlands. Partner organisations: Novartis Pharma AG; University of Southampton, Southampton, UK; Academic Medical Centre, University of Amsterdam, Amsterdam, The Netherlands; Imperial College London, London, UK; University of Catania, Catania, Italy; University of Rome "Tor Vergata", Rome, Italy; Hvidore Hospital, Hvidore, Denmark; Jagiellonian Univ. Medi. College, Krakow, Poland; University Hospital, Inselspital, Bern, Switzerland; Semmelweis University, Budapest, Hungary; University of Manchester, Manchester, UK; Université d'Aix-Marseille, Marseille, France; Fraunhofer Institute, Hannover, Germany; University Hospital, Umea, Sweden; Ghent University, Ghent, Belgium; Ctr. Nat. Recherche Scientifique, Lyon, France; Università Cattolica del Sacro Cuore, Rome, Italy; University Hospital, Copenhagen, Denmark; Karolinska Institutet, Stockholm, Sweden; Nottingham University Hospital, Nottingham, UK; University of Bergen, Bergen, Norway; Netherlands Asthma Foundation, Leusden, The Netherlands; European Lung Foundation, Sheffield, UK; Asthma UK, London, UK; European. Fed. of Allergy and Airways Diseases Patients' Associations, Brussels, Belgium; Lega Italiano Anti Fumo, Catania, Italy; International Primary Care Respiratory Group, Aberdeen, UK; Philips Research Laboratories, Eindhoven, The Netherlands; Synairgen Research Ltd, Southampton, UK; Aerocrine $\mathrm{AB}$, Stockholm, Sweden; BioSci Consulting, Maasmechelen, Belgium; Almirall; AstraZeneca; Boehringer Ingelheim; Chiesi; GlaxoSmithKline; Roche; UCB; Janssen Biologics BV; Amgen NV; Merck Sharp \& Dome Corp. Members of the ethics board: J-B. Prins, biomedical research, LUMC, The Netherlands; M. Gahlemann, clinical care, BI, Germany; L. Visintin, legal affairs, LIAF, Italy; H. Evans, paediatric care, Southampton, UK; M. Puhl, patient representation (co-chair), NAF, The Netherlands; L. Buzermaniene, patient representation, EFA, Lithuania; V. Hudson, patient representation, Asthma UK; L. Bond, patient representation, Asthma UK; P. de Boer, patient representation and pathobiology, IND; G. Widdershoven, research ethics, VUMC, The Netherlands; R. Sigmund, research methodology and biostatistics, BI, Germany. The patient input platform: A. Roberts, UK; D. Supple (chair), UK; D. Hamerlijnck, The Netherlands; J. Negus, UK; J. Kamphuis, The Netherlands; L. Sergison, UK; L. Visintin, Italy; P. de Boer (co-chair), The Netherlands; S. Onstein, The Netherlands. Members of the safety monitoring board: W. MacNee, clinical care; R. Bernardini, clinical pharmacology; Louis Bont, paediatric care and infectious diseases; P-A. Wecksell, patient representation; P. de Boer, patient representation and pathobiology (chair); M. Gahlemann, patient safety advice and clinical care (co-chair); R. Sigmund, bio-informatician.

Support statement: U-BIOPRED is supported through an Innovative Medicines Initiative Joint Undertaking under grant agreement number 115010, resources of which are composed of financial contribution from the European Union's Seventh Framework Program (FP7/2007-2013) and European Federation of Pharmaceutical Industries and Associations companies' in-kind contribution (www.imi.europa.eu). J.M. Perotin was supported by the European Respiratory Society (fellowship LTRF 2017), Association Régionale pour l'Aide aux Insuffisants Respiratoires de Champagne-Ardenne, Association Nationale de Formation Continue en Allergologie, Association des Allergologues de Champagne-Ardenne and Association des Pneumologues de Champagne-Ardenne. Funding information for this article has been deposited with the Crossref Funder Registry. 
Author contributions: Substantial contributions to the conception or design of the work: J.M. Perotin, J.P.R. Schofield, D.E. Davies and R. Djukanović. Acquisition, analysis, or interpretation of data: J.M. Perotin, J.P.R. Schofield, J. Brandsma, F. Strazzeri, A. Bansal, X. Yang, A. Rowe, J. Corfield, S.J. Wilson, J. Ward, R. Lutter, D.E. Shaw, P.S. Bakke, M. Caruso, B. Dahlén, S.J. Fowler, I. Horváth, P. Howarth, N. Krug, P. Montuschi, M. Sanak, T. Sandström, K. Sun I. Pandis, C. Auffray, B. De Meulder, D. Lefaudeux, J.H. Riley, A.R. Sousa, S-E. Dahlen, I.M. Adcock, K.F. Chung, P.J. Sterk, P.J. Skipp, D.E. Davies and R. Djukanović. Drafting the manuscript or revising it critically for important intellectual content: J-M. Perotin, J.P.R. Schofield, J.E. Collins, D.E. Davies and R. Djukanović. Final approval of the manuscript version to be published: all authors.

Conflict of interest: J.M. Perotin has nothing to disclose. J.P.R. Schofield has nothing to disclose. S.J. Wilson has nothing to disclose. J. Ward has nothing to disclose. J. Brandsma has nothing to disclose. F. Strazzeri has nothing to disclose. A. Bansal has nothing to disclose. X. Yang has nothing to disclose. A. Rowe is a full-time employee and shareholder of Janssen Pharmaceutical Companies of Johnson and Johnson. J. Corfield has nothing to disclose. R. Lutter has nothing to disclose. D.E. Shaw reports fees for lectures at company sponsored symposia and support to attend an international conference from AstraZeneca, fees for lectures at company sponsored symposia and investigator-initiated research funding from Boehringer Ingelheim, fees for lectures at company sponsored symposia from Novartis and Teva, a fee for presentation from Circassia, and a grant for an investigator led study from GSK, outside the submitted work. P.S. Bakke reports personal fees from GSK, AZ, Novartis andTeva, outside the submitted work. M. Caruso has no conflict of interest to disclose. B. Dahlén has nothing to disclose. S.J. Fowler reports personal fees and non-financial support from AstraZeneca, grants and personal fees from Boehringer Ingelheim, and personal fees from Novartis and Teva, outside the submitted work. I. Horváth reports personal fees from Astra Zeneca, Boehringer Ingelheim, Novartis, CSL, Chiesi, Roche, GSK, Berlin-Chemie and Sandoz, outside the submitted work. P. Howarth reports personal fees from GSK, outside the submitted work. N. Krug has nothing to disclose. P. Montuschi has nothing to disclose. M. Sanak has nothing to disclose. T. Sandström reports Fees for lectures and course arrangement from Boehringer Ingelheim, outside the submitted work. K. Sun has nothing to disclose. I. Pandis has nothing to disclose. C. Auffray reports grants from Innovative Medicine Initiative, during the conduct of the study. B. De Meulder reports grants from Innovative Medicine Initiative, during the conduct of the study. D. Lefaudeux reports grants from Innovative Medicine Initiative, grants from Innovative Medicine Initiative, during the conduct of the study. J.H. Riley reports that he has shares in and is employed by GSK. A.R. Sousa has nothing to disclose. S-E. Dahlén has nothing to disclose. I.M. Adcock reports grants from EU-IMI, during the conduct of the study. K.F. Chung has received honoraria for participating in Advisory Board meetings of GSK, AZ, BI, Teva, Novartis and Merck regarding treatments for asthma and chronic obstructive pulmonary disease, and has also been renumerated for speaking engagements. P.J. Sterk reports grants from Innovative Medicines Initiative, during the conduct of the study. P.J. Skipp has nothing to disclose. J.E. Collins reports a patent application for use of a genetically modified Drosophila line carrying one or more mammalian genes associated with a chronic respiratory disease and uses to screen the impact of such genes. D.E. Davies has nothing to disclose. R. Djukanović reports receiving fees for lectures at symposia organised by Novartis, AstraZeneca and TEVA, consultation for TEVA and Novartis as member of advisory boards, and participation in a scientific discussion about asthma organised by GlaxoSmithKline. He is a co-founder and current consultant, and has shares in Synairgen, a University of Southampton spin out company.

\section{References}

1 Tariq K, Schofield JPR, Nicholas BL, et al. Sputum proteomic signature of gastro-oesophageal reflux in patients with severe asthma. Respir Med 2019; 150: 66-73.

2 Shaw DE, Sousa AR, Fowler SJ, et al. Clinical and inflammatory characteristics of the European U-BIOPRED adult severe asthma cohort. Eur Respir J 2015; 46: 1308-1321.

3 Larry W. Topological data analysis. Ann Rev Stat Appl 2018; 5: 501-532.

4 Baarsma HA, Königshoff M. WNT signalling in chronic lung diseases. Thorax 2017; 72: 746-759.

5 Yiew NKH, Chatterjee TK, Tang YL, et al. A novel role for the Wnt inhibitor APCDD1 in adipocyte differentiation: implications for diet-induced obesity. J Biol Chem 2017; 292: 6312-6324.

6 Wammers M, Schupp AK, Bode JG, et al. Reprogramming of pro-inflammatory human macrophages to an anti-inflammatory phenotype by bile acids. Sci Rep 2018; 8: 255.

7 Liu J, Huang N, Li N, et al. 2-(1H-Benzimidazol-2-yl)-4,5,6,7-tetrahydro-2H-indazol-3-ol, a benzimidazole derivative, inhibits $\mathrm{T}$ cell proliferation involving $\mathrm{H}^{+} / \mathrm{K}^{+}$-ATPase inhibition. Molecules 2014; 19: 17173-17186.

8 Namazi MR, Jowkar F. A succinct review of the general and immunological pharmacologic effects of proton pump inhibitors. J Clin Pharm Ther 2008; 33: 215-217.

9 Chun J, Prince A. Ca ${ }^{2+}$ signaling in airway epithelial cells facilitates leukocyte recruitment and transepithelial migration. J Leukoc Biol 2009; 86: 1135-1144.

10 Waser M, Mesaeli N, Spencer C, et al. Regulation of calreticulin gene expression by calcium. J Cell Biol 1997; 138: $547-557$.

11 Schillinger W, Teucher N, Sossalla S, et al. Negative inotropy of the gastric proton pump inhibitor pantoprazole in myocardium from humans and rabbits: evaluation of mechanisms. Circulation 2007; 116: 57-66.

12 Liu Y, Chen GY, Zheng P. CD24-Siglec G/10 discriminates danger from pathogen-associated molecular patterns. Trends Immunol 2009; 30: 557-561.

13 Ye P, Nadkarni MA, Simonian M, et al. CD24 regulated gene expression and distribution of tight junction proteins is associated with altered barrier function in oral epithelial monolayers. BMC Cell Biol 2009; 10: 2 .

14 Fairbridge NA, Southall TM, Ayre DC, et al. Loss of CD24 in mice leads to metabolic dysfunctions and a reduction in white adipocyte tissue. PLoS One 2015; 10: e0141966.

15 Duchartre Y, Kim YM, Kahn M. The Wnt signaling pathway in cancer. Crit Rev Oncol Hematol 2016; 99: $141-149$. 